REVISTA DE GEOCIÊNCIAS DO NORDESTE

Northeast Geosciences Journal

v. $6, \mathrm{n}^{\circ} 2(2020)$

ISSN: $2447-3359$

https://doi.org/10.21680/2447-3359.2020v6n2ID20544

\title{
DESEMPENHO DE PLACAS DE FLUXO DE CALOR NO SOLO EM ÁREA COM CAPIM BRAQUIÁRIA
}

\begin{abstract}
João Paulo Chaves Couto ${ }^{1}$; Aureo Silva de Oliveira $^{2}$; Tatyana Keyty de Souza Borges ${ }^{3}$; Monikuelly Mourato Pereira ${ }^{4}$
\end{abstract}

${ }^{1}$ Mestre em Engenharia Agrícola, Núcleo de Engenharia de Água e Solo, Universidade Federal do Recôncavo da Bahia (UFRB), Cruz das Almas/BA, Brasil.

ORCID: https://orcid.org/0000-0002-8859-3271

Email: jpauloengagro@gmail.com

${ }^{2}$ Doutor em Engenharia Agrícola e de Biossistemas, Núcleo de Engenharia de Água e Solo, Universidade Federal do Recôncavo da Bahia (UFRB), Cruz das Almas/BA, Brasil.

ORCID: https://orcid.org/0000-0001-7661-551X

Email: aureo@ufrb.edu.br

${ }^{3}$ Doutora em Engenharia Agrícola, Núcelo de Engenharia de Água e Solo, Universidade Federal do Recôncavo da Bahia (UFRB), Cruz das Almas/BA, Brasil.

ORCID: https://orcid.org/0000-0002-1700-4150

Email: tatyana_keyty@yahoo.com.br

${ }^{4}$ Doutora em Engenharia Agrícola, Núcelo de Engenharia de Água e Solo, Universidade Federal do Recôncavo da Bahia (UFRB), Cruz das Almas/BA, Brasil.

ORCID: https://orcid.org/0000-0003-4974-7570

Email: monikuelly@hotmail.com

\section{Resumo}

As placas de fluxo de calor no solo são utilizadas em estudos de balanço de energia e demanda hídrica. Objetivou-se, com este estudo avaliar dois diferentes modelos de placas de fluxo de calor no solo, HFT3.1 e HFP01, denominadas REBS e HUKS para período de solo seco na Universidade Federal do Recôncavo da Bahia - UFRB, Campus Cruz das Almas, entre outubro e dezembro de 2015. Os valores $\mathrm{R}^{2}$, para o período de solo seco, foram de 99,34; 96,86 e 99,04\% comparando placas do modelo HFT3.1 (REBS1 x REBS2), do modelo HFP01 (HUKS1 x HUKS2) e entre os modelos REBS e HUKS respectivamente. O modelo REBS apresentou maior amplitude de medição em relação ao modelo HUKS, sendo que a diferença pode ser atribuída às condições de instalação das placas.
Palavras-chave: Balanço de energia; Modelos de placas; HFT3.1; HFP01.

\section{PERFORMANCE OF SOIL HEAT FLUX PLATES IN A FIELD COVERED WITH BRACHIARIA GRASS AREA}

\begin{abstract}
Soil heat flux plates are used in studies of energy balance and water demand. The objective of this study was to evaluate two different models of heat flux plates in the soil, HFT3.1 and HFP01, called REBS and HUKS for dry soil season at the Federal University of Recôncavo da Bahia - UFRB, Campus Cruz das Almas, between October and December 2015. $\mathrm{R}^{2}$ values were 99.34; 96.86 and $99.04 \%$ comparing plates of the model HFT3.1 (REBS1 x REBS2), of the model HFP01 (HUKS1 x HUKS2) and between the models REBS and HUKS respectively. The REBS model presented a greater measurement range in relation to the HUKS model, the difference can be attributed to the installation conditions of the plates.
\end{abstract}

Keywords: Energy balance; Soil heat flux; HFT3.1; HFP01.

\section{RENDIMIENTO DE LAS PLACAS DE FLUJO DE CALOR DEL SUELO EM UM ÁREA DE HIERBA BRACHIARIA}

\section{Resumen}

Las placas de flujo de calor del suelo se utilizan en estudios de equilibrio energético y demanda de agua. El objetivo de este estudio fue evaluar dos modelos diferentes de placas de flujo de calor en el suelo, HFT3.1 y HFP01, llamados REBS y HUKS para la temporada de suelo seco en la Universidad Federal de Recôncavo da Bahia - UFRB, Campus Cruz das Almas, entre Octubre y diciembre de 2015. Los valores de $\mathrm{R}^{2}$ fueron 99.34; 96.86 y $99.04 \%$ comparando placas del modelo HFT3.1 (REBS1 x REBS2), del modelo HFP01 (HUKS1 x HUKS2) y entre los modelos REBS y HUKS respectivamente. El modelo REBS presentó un mayor rango de medición en relación con el modelo HUKS, la diferencia puede atribuirse a las condiciones de instalación de las placas.

Palabras-clave: Balance energético; Modelos de tablero; HFT3.1; HFP01. 


\section{INTRODUÇÃO}

O fluxo de calor no solo $(\mathrm{G})$ é um componente da radiação líquida $(\mathrm{Rn})$ e representa a quantidade de energia utilizada no processo de variação térmica do solo. Para Soares (2013), G representa a fração de $\mathrm{Rn}$ transferida a níveis inferiores do solo, representando a energia radiante absorvida ou liberada na superfície no intervalo de tempo (PAYERO et al., 2005). Sua quantificação é necessária para determinação de fluxos de calor latente e sensível (GALVANI et al., 2001) no balanço de energia.

Conforme Moura e Querino (2010), G ocorre por condução devido desequilíbrio térmico do sistema durante o dia ou à noite, caracterizando as trocas de energia entre superfície e atmosfera. Assim, estudos micrometeorológicos visam estimar trocas de energia e matéria em áreas naturais ou modificadas (GALEANO et al., 2013).

Segundo Carneiro et al. (2013) G é dependente das propriedades físicas do solo, envolvendo condutividade e difusividade térmica, bem como a capacidade calórica. Para Sauer e Horton (2005), muitos estudos, envolvendo densidade de fluxo de calor no solo, usaram placas de fluxo de calor. Estas possuem propriedades térmicas definidas e constantes que medem a densidade do fluxo de calor proporcional ao fluxo de calor no solo.

As placas resistem a longo prazo de uso no campo, suportando a instalação repetida e escavação, permitindo medições frequentes e precisas (OCHSNER et al., 2006).

Há diferentes modelos de placas, no entanto, cada um possui desempenho diferente, devido às distintas características físicas, bem como fator de calibração; havendo, ainda, placas autocalibrantes.

Diante do que foi exposto, objetivou-se comparar medições de fluxo de calor no solo a partir de dois modelos de placas de fluxo de calor sob condições variáveis de grau de cobertura da superfície do solo.

\section{METODOLOGIA}

O experimento foi conduzido na Universidade Federal do Recôncavo da Bahia (UFRB), no município de Cruz das Almas BA (1240'39" S; 3906'23" W; $225 \mathrm{~m}$ ) em um intervalo temporal compreendido entre outubro e dezembro de 2015.

Segundo Silva et al. (2016) a região possui duas classificações climáticas, sendo a primeira proposta por Köeppen Ometto do tipo Ami (chuva anual dez vezes maior que o mês mais seco) e a segunda conforme a metodologia de Thornthwaite é C1dA'a' classificada como $\mathrm{C} 1$ (seco e subúmido) com pluviosidade média anual de $1131,2 \mathrm{~mm}$, e médias anuais que variam em torno de $81 \%$ e $23,9{ }^{\circ} \mathrm{C}$ para umidade relativa temperatura do ar respectivamente. A área experimental foi de $1764 \mathrm{~m}^{2}$, ocupada em sua totalidade por pastagem de capim braquiária (Brachiaria decumbens, L.), sob grau de cobertura variável sob condições de sequeiro durante o período de outubro a dezembro de 2015.

Ao longo do estudo, foram realizadas medidas através de uma estação meteorológica automática localizada na área de estudo com instrumentos conectados a uma unidade acumuladora de dados (datalogger), modelo CR1000 (Campbell Scientifc, Logan, Utah, USA), sendo, então, armazenados em médias horárias e totais horários.
Para registro do saldo de radiação ( $\mathrm{Rn}$ ) foi utilizado um saldo radiômetro modelo CNR4 (Kipp \& Zonen, The Netherlands), instalado a $1,5 \mathrm{~m}$ de altura. Para registro da precipitação pluviométrica utilizou-se um pluviômetro modelo TE525MM (Texas Electronics, Dallas, Texas, USA) instalado com área de captação a $0,5 \mathrm{~m}$ da superfície do solo.

A temperatura e umidade relativa do ar foi medida por um temohigrômetro modelo HMP60 (Vaisala, Inc. Helsinki, Finland).

A velocidade e direção do vento foram medidas por meio de um anemômetro de canecas, modelo 03001 (RM Young, inc., USA) instalado a $3 \mathrm{~m}$ de altura da superfície.

Neste estudo, foram coletados dados de fluxo de calor no solo através de quatro placas, as quais foram instaladas paralelamente à superfície do solo a uma profundidade de $0,08 \mathrm{~m}$ distribuídas em quatro pontos distintos. Em duas das parcelas (P1 e P2), utilizou-se as placas do modelo HFT3.1 (Radiation and Energy Balance Systems, Seattle, Washington, USA) (doravante REBS1 e REBS2) contra duas placas do modelo HFP01 (Hukseflux Thermal Systems, Delft, The Netherlands) (doravante identificadas como HUKS1 e HUKS2) instaladas nas parcelas P3 e P4.

Dois termopares instalados ao lado da placa a 0,02 e 0,06 m abaixo da superfície do solo e uma sonda de TDR (Reflectometria no domínio do tempo) instalada entre 0,03 e $0,06 \mathrm{~m}$ de profundidade para monitoramento da umidade do solo $(\theta)$.

A determinação do fluxo de calor na superfície do solo foi calculada, conforme Kustas et al. (2000), representada pela Equação 1:

$$
\mathrm{G}_{0}=\mathrm{G}_{8}+\frac{\left(\mathrm{T}_{\mathrm{i}} \mathrm{T}_{\mathrm{i} 1}\right) \mathrm{C}_{\mathrm{s}} \quad \mathrm{z}}{\Delta \mathrm{t}}
$$

Em que,

$\mathrm{G}_{0}$ - fluxo de calor na superfície do solo $\left(\mathrm{W} \mathrm{m}^{-2}\right)$;

$\mathrm{G}_{8}$ - fluxo de calor no solo medido a $0,08 \mathrm{~m}$ profundidade $\left(\mathrm{W} \mathrm{m}^{-}\right.$ $\left.{ }^{2}\right)$;

$\mathrm{T}_{\mathrm{i}}$ - temperatura do solo $\left({ }^{\circ} \mathrm{C}\right)$ no instante $\mathrm{i}$;

$\mathrm{T}_{\mathrm{i}-1}$ - temperatura do solo $\left({ }^{\circ} \mathrm{C}\right)$ no instante anterior;

$\mathrm{C}_{\mathrm{s}}$ - capacidade calórica do solo úmido $\left(\mathrm{MJ} \mathrm{m}^{-3}{ }^{\circ} \mathrm{C}^{-1}\right)$;

$\mathrm{z}$ - profundidade de instalação da placa $(\mathrm{m})$;

$\Delta \mathrm{t}$ - intervalo de agrupamento dos dados (s).

O monitoramento do grau de cobertura do solo foi realizado através do processamento de imagens digitais via software Image ${ }^{\circledR} 1.48 \mathrm{v}$, iniciando sob grau de cobertura zero, ou seja, solo nu até $100 \%$ de cobertura. Assim, a vegetação foi ceifada e as fotografias foram capturadas em um intervalo de três dias em dez pontos escolhidos aleatoriamente, incluindo os quatro referentes aos locais de instalação das placas.

Para avaliação comparativa entre placas de fluxo de calor no solo, foi realizada uma análise de regressão, levando em consideração a comparação entre sensores de mesmo fabricante (REBS1 x REBS2; HUKS1 x HUKS2) e sensores de fabricantes diferentes (REBS x HUKS), sendo os valores obtidos através das médias de cada modelo e realizados as correlações existentes.

A comparação entre os modelos de placas de fluxo de calor utilizados neste estudo foi realizada considerando um período seco (outubro a dezembro), bem como as diferentes condições do 
grau de cobertura da superfície do solo proposto pela vegetação local (capim braquiária).

A dispersão entre os valores medidos pelos fluxímetros foi evidenciada através do erro padrão da estimativa (SEE), extraindo da ANOVA da regressão os coeficientes dos modelos de regressão e os respectivos testes estatísticos de significância para os modelos.

\section{RESULTADOS E DISCUSSÃO}

O regime pluviométrico está relacionado aos processos de ordem química, física e biológica na superfície terrestre, o qual altera as características hídricas e térmicas do solo, proporcionando influência direta na determinação do fluxo de calor no mesmo.

Ao longo do estudo, foi registrada uma precipitação pluviométrica de 19,3 $\mathrm{mm}$, no período de estiagem (outubro a dezembro).

O solo da área experimental foi classificado como de textura areia franca. A determinação da densidade do solo foi realizada pelo método do anel volumétrico, a partir de amostras de solo coletadas na área com densidade média do solo de $1,75 \mathrm{~kg} \mathrm{~m}^{-3}$.

Os valores diários de umidade do solo, em base de volume durante todo o período, podem ser insignificantes, uma vez que os valores de $\theta_{\mathrm{v}}$, medidos através da TDR a uma profundidade entre 3 e $6 \mathrm{~cm}$, foram relativamente pequenos (1 a 1,5\%), apresentando pouca variação durante 0 estudo. Este comportamento foi também evidenciado por Peng et al. (2015) ao posicionarem sondas de TDR a 0,02 $\mathrm{m}$ de profundidade. Para os autores, isso ocorre devido à existência de grandes gradientes de concentração de vapor próximo da interface solo-atmosfera e um espaço poroso cheio de ar está disponível para difusão de vapor.

Salienta-se que, durante a condução do experimento, a temperatura média do ar observada foi de $25,1^{\circ} \mathrm{C}$, com variações de 18,5 a $31,9^{\circ} \mathrm{C}$. A média da umidade relativa do ar foi de $76 \%$ ao longo do período em estudo, com mínima de $56 \%$ e máxima de $97 \%$ e da velocidade média do vento foi de $2,1 \mathrm{~m} \mathrm{~s}-1$, chegando a atingir pico máximo de $5,8 \mathrm{~m} \mathrm{~s}^{-1}$.

A Figura 1 mostra uma análise temporal do fluxo de calor no solo $\left(\mathrm{G}_{8}\right.$ e $\left.\mathrm{G}_{0}\right)$ dos valores médios para cada modelo, sob condição de solo seco entre o DJ 328 e DJ 333.

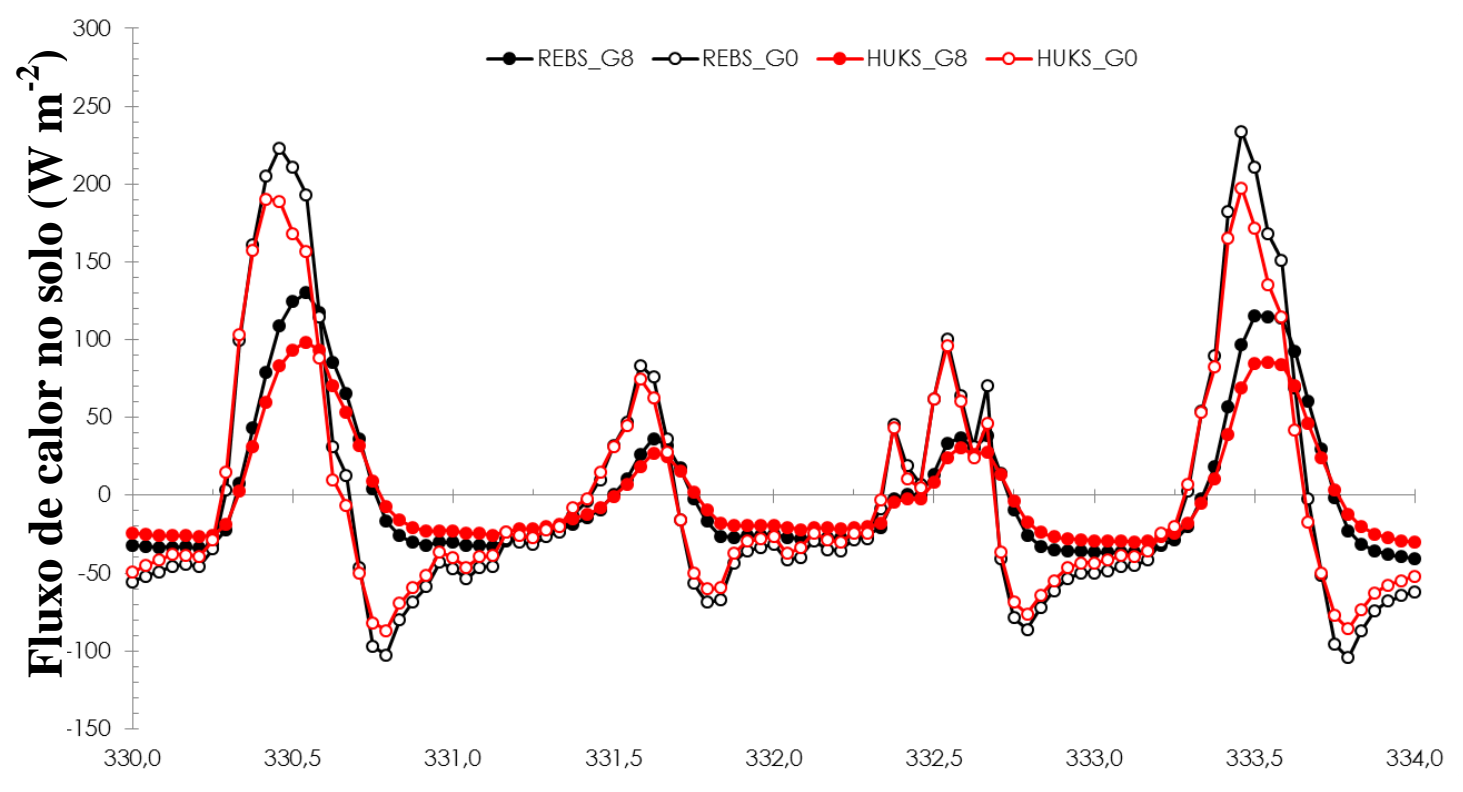

\section{Tempo (Dia Juliano)}

Figura 1 - Evolução do fluxo de calor no solo médio $\left(G_{8}\right.$ e $\left.G_{0}\right)$ com as placas HFT3.1 (REBS) E HFP01 (HUKS) entre 24 e 29/10/2015 para período de solo seco em Cruz das Almas, Bahia. Fonte:Autor.

Nota-se, na evolução do fluxo de calor no solo, tanto para $\mathrm{G}_{8}$ quanto para $\mathrm{G}_{0}$, uma amplitude maior em medidas obtidas a partir das leituras das placas modelo HFT3.1 (REBS) em relação às placas modelo HFP01 (HUKS) para valores agrupados em médias horárias.

Verifica-se que, devido à baixa umidade do solo, ocorreu predominância de valores positivos durante maior parte do dia. Como se sabe, o solo com baixa umidade permite maior amplitude térmica devido ao elevado calor específico da água. Desta forma, o baixo teor de umidade favoreceu maior variação da temperatura do solo, resultando em valores mais elevados de fluxo de calor no solo medido através das placas, bem como nos valores corrigidos para a superfície do solo.

Para a Figura 1, as médias de $\mathrm{G}_{8}$ e $\mathrm{G}_{0}$ apresentaram um curso diário uniformemente distribuído. No entanto, é possível perceber a ocorrência de valores reduzidos nas medições do fluxo de calor 
no solo $\left(\mathrm{G}_{8}\right.$ e $\left.\mathrm{G}_{0}\right)$ aos DJ 331 e DJ 332. Essa redução dos valores se deve ao fato da presença de dias com alta nebulosidade, em que foi observada transmissividade atmosférica de 0,25 e 0,31 , respectivamente.

Vale ressaltar que a alta nebulosidade provocou interceptação parcial da radiação solar que chega à superfície da terra, que influenciou na redução da temperatura do solo e, consequentemente, na redução de amplitudes de $\mathrm{G}_{8}$ e $\mathrm{G}_{0}$, a qual reforça a influência da cobertura das nuvens no comportamento da transferência de energia no solo.

O grau de cobertura do solo foi variável para o período avaliado apresentado no gráfico, sendo que, para o intervalo compreendido entre o DJ 330 ao DJ 333, o grau de cobertura proporcionado pela pastagem apresentou pequena variação $(23,93$ a $24,36 \%)$.

Correlacionando os dados horários obtidos por cada modelo de placa de fluxo de calor no solo de mesmo fabricante (REBS e HUKS), o modelo matemático encontrado que melhor ajustou os dados foi o linear. A Tabela 1 resume os indicadores de desempenho dos modelos avaliados para todas as combinações possíveis das placas de fluxo de calor para valores de $\mathrm{G}_{8}$ e $\mathrm{G}_{0}$.

Tabela 1 - Coeficientes de regressão linear $\left(a, W^{-2} / W^{-2}\right.$ e b, $\left.W m^{-2}\right)$, coeficiente de determinação $\left(R^{2}\right)$, e erro padrão da estimativa (SEE, $\mathrm{Wm}^{-2}$ ) para todas as combinações avaliadas de comparação de placas de fluxo de calor. Fonte: autor.

\begin{tabular}{|c|c|c|c|c|}
\hline & \multicolumn{4}{|c|}{$\mathbf{G}_{8}$} \\
\hline Confronto entre placas & $\mathbf{A}$ & b & $\mathbf{R}^{2}$ & SEE \\
\hline REBS1 x REBS2 & 0,85 & 4,55 & 0,98 & 7,83 \\
\hline HUKS1 x HUKS2 & 1,12 & 2,88 & 0,97 & 7,89 \\
\hline REBS1 x HUKS1 & 0,65 & $-0,42$ & 0,97 & 6,13 \\
\hline REBS1 x HUKS2 & 0,64 & 2,61 & 0,99 & 6,02 \\
\hline REBS2 x HUKS1 & 0,84 & $-3,27$ & 0,98 & 7,03 \\
\hline REBS2 x HUKS2 & 0,83 & $-0,17$ & 0,99 & 2,86 \\
\hline \multirow[t]{2}{*}{ REBS $x$ HUKS } & 0,73 & $-0,07$ & 0,99 & 3,31 \\
\hline & \multicolumn{4}{|c|}{$\mathbf{G}_{\mathbf{0}}$} \\
\hline Confronto entre placas & A & b & $\mathbf{R}^{2}$ & SEE \\
\hline REBS1 x REBS2 & 0,92 & 3,54 & 0,98 & 11,45 \\
\hline HUKS1 x HUKS2 & 0,91 & 3,94 & 0,93 & 21,32 \\
\hline REBS1 x HUKS1 & 0,82 & $-1,35$ & 0,96 & 15,48 \\
\hline REBS1 x HUKS2 & 0,79 & 2,41 & 0,98 & 10,44 \\
\hline REBS2 x HUKS1 & 0,87 & $-4,36$ & 0,95 & 18,88 \\
\hline REBS2 x HUKS2 & 0,85 & $-0,58$ & 0,99 & 7,02 \\
\hline REBS $x$ HUKS & 0,83 & $-0,95$ & 0,99 & 6,33 \\
\hline
\end{tabular}

Os modelos encontrados são válidos para simulação do fluxo de calor no solo ( $\mathrm{G}_{8}$ e $\mathrm{G}_{0}$ ) para HUKS, baseado em valores obtido de REBS, uma vez que todas as combinações apresentaram elevados coeficientes de determinação $\left(\mathrm{R}^{2}\right)$ com modelo linear do tipo $\mathrm{Y}=\mathrm{ax}+\mathrm{b}$. No entanto, é perceptível que, para os valores médios de REBS versus HUKS, os valores do erro padrão da estimativa são menores.

Para avaliação do fluxo de calor na interface solo-atmosfera, é possível notar que os valores obtidos, a partir da estimativa de $\mathrm{G}_{0}$ para as duas placas, apresentam alta correlação $\left(\mathrm{R}^{2}=0,991\right)$, o que permite estimar $\mathrm{G}_{0}$ para o modelo HUKS baseado em valores de $\mathrm{G}_{0}$ estimados por REBS com confiabilidade de $99,19 \%$ do modelo gerado.

Uma avaliação foi realizada levando em consideração a média dos valores horários do fluxo de calor no solo para os modelos HFT3.1 (REBS) e HFP01 (HUKS) nos períodos diurno e noturno ao longo do estudo em que foi possível verificar as diferenças nos valores médios ( $\mathrm{W} \mathrm{m}^{-2}$ ) de REBS (HFT3.1) durante o período diurno e noturno, em relação ao fluxo HUKS (HFP01).

Percentualmente, durante o período diurno, a diferença entre os modelos foi de $28,14 \%$ de superestimativa nos valores da placa do modelo REBS em relação à placa do modelo HUKS. Resultado semelhante foi observado durante o período noturno, ou seja, foi observado uma superestimativa na ordem de $29,22 \%$.

Uma superestimativa para REBS (HFT3.1) foi também observada por Sauer et al. (2008), realizando testes em campo e em laboratório, comparando-a com um novo design da placa de fluxo de calor (placa perfurada). As diferenças observadas podem ser atribuídas devido à sensibilidade de posicionamento das placas, bem como as características locais, visto que as placas foram instaladas em locais distintos e, portanto, propícias às alterações.

É possível que a escolha de um dos modelos de placas testado neste estudo impactará o fechamento do balanço de energia da superfície analisada, visto que haverá sub ou superestimativa da energia disponível (Rn-G) para os fluxos turbulentos de calor sensível $(\mathrm{H})$ e calor latente $(\mathrm{H})$.

Durante a instalação das placas podem ocorrer diversos erros, visto que pode acumular partículas na parte sensível à temperatura da placa, podendo variar, significativamente, as medições do fluxo de calor. Além disso, Weber et al. (2007) afirmam que erros significativos, em medidas de fluxo de calor no solo, através da utilização de fluxímetros, podem ocorrer quando decorrente de fluxos de calor em substratos porosos grosseiro.

Weber et at. (2007), ao testarem o desempenho de placas de fluxo de calor no solo do modelo HUKS, encontraram valores mais elevados quando comparados com o método do gradiente de temperatura em laboratório, enquanto que, sob condições de campo, foi observado uma subestimativa de $26 \%$, em que os autores implicam esse comportamento devido à sensibilidade de instalação do fluxímetro. Peng et al (2015), avaliando a performance de HUKS, obtiveram uma superestimativa pela placa em comparação com o método gradiente a uma profundidade de $0,02 \mathrm{~m}$, no entanto, nas profundidades de $0,06 \mathrm{e}$ $0,10 \mathrm{~m}$, observaram uma subestimativa nos valores medidos. 


\section{CONSIDERAÇÕES FINAIS}

O fluxo de calor no solo medido pela placa do modelo HFT3.1 (REBS) apresenta maiores amplitudes quando comparados com o modelo HFP01 (HUKS). A escolha de um dos modelos pode impactar o fechamento do balanço de energia local sub ou superestimando a energia disponível para os fluxos turbulentos de calor sensível $(\mathrm{H})$ e calor latente $(\lambda \mathrm{ET})$.

\section{REFERÊNCIAS}

BASTABLE, H. G.; SHUTTLEWORTH, W. J.; DALLAROSA, R. L. G.; FISCH, G.; NOBRE, C.A. Observations of climate, albedo and surface radiation over cleared and undisturbed Amazonian Forest. International Journal of Climatology, v.13, n.7, 783- 796, 1993.

CARNEIRO, R. G.; MOURA, M. A. L.; LYRA, R. F. F.; ANDRADE, A. M. D.; SANTOS, A. B.; MAIA, R. G. X. Fluxo de calor do solo e saldo de radiação dentro de uma área de mata atlântica em comparação com uma área de cana-de-açúcar. Revista Brasileira de Geografia Física, v.6, n.3, 555-565, 2013.

FUNARI, F. L.; PEREIRA FILHO, A. J. Estimativa do fluxo de calor no solo a partir da temperatura do solo em São Paulo, SP. Revista do Instituto Geológico, v.38, n.1, 49-57, 2017.

GALEANO, D. C., BIUDES, M. S., DANELICHEN, V. H. M., FABIAN, F. A., SOUZA, M. C. Energy balance in a pasture in the Pantanal. Journal of Biotechnology and Biodiversity, v. 4, n.2, 113-118, 2013.

GALVANI, E., ESCOBEDO, J. F., PEREIRA, A. B. Balanço de radiação e fluxo de calor no solo em ambiente natural e protegido cultivado. Bragantia,v. 60, n.2, 139-147, 2001.

KUSTAS, W. P., PRUEGER, J. H., HATFIELD, J. L., RAMALINGAM, K., HIPPS, L. E. Variability in soil heat flux from a mesquite dune site. Agricultural and Forest Meteorology, v.103, n.3, 249-264, 2000.

MOURA, M. A. L., QUERINO, C. A. S. Variação sazonal do fluxo de calor no solo dentro de um manguezal tropical. Revista Brasileira de Engenharia Agrícola e Ambiental, v.14, n.2, 296-302, 2010.

OCHSNER, T. E., SAUER, T. J., HORTON, R. Field tests of the soil heat flux plate method and some alternatives. Agronomy journal, v.98, n.4, 1005-1014, 2006.

PAYERO, J. O., NEALE, C. M. U., WRIGHT, J. L. Estimating soil heat flux for alfalfa and clipped tall fescue grass. American Society of Agricultural Engineers, v.21, n.3, 401409, 2005.

PENG, X., HeITMAN, J., HORTON, R., REN, T. Field evaluation and improvement of the plate method for measuringsoil heat flux density. Agricultural and Forest Meteorology, v.214-215, 341-349, 2015.
PRIANTE FILHO, N.; VOURLITIS, G. L.; HAYASHI, M. M. S.; NOGUEIRA, J. S.; CAMPELO JÚNIOR, J. H.; NUNES, P. C.; SOUZA, L. S.; COUTO, E. G.; HOEGER, W.; RAITER, F.; TRIENWEILER, J. L.; MIRANDA, E. J.; PRIANTE, P. C.; FRITZEN, C. L.; LACERDA, M.; PEREIRA, L. C.; BIUDES, M. S.; SULI, G. S.; SHIRAIWA, S.; PAULO, S. R.; SILVEIRA, M. Comparison of the mass and energy Exchange of a pasture and a mature transitional tropical Forest of the southern Amazon Basin durin a seasonal transition. Global Change Biology, v.10, n.5, 863-876, 2004.

SÁ, L. D. A.; VISWANADHAM, Y.; MANZI, A. O. Energy flux partitioning over the Amazon Forest. Theoretical and Applied Climatology, v.39, n,1, 1-16, 1988.

SAUER, T. J., AKINYEMI, O. D., THERY, P., HEITMAN, J. L., DESUTTER, T. M., HORTON, R. Evaluation of a new, perforated heat flux plate design. International Communications in Heat and Mass Transfer, v.35, n.7, 800804, 2008.

SAUER, T. J., HORTON, R. 2005. Soil heat flux. In: Hatfield J. L., Baker J. M., editors. Micrometeorology in agricultural systems. Agron Monogr, 47. Am Soc of Agron Madison, WI. p.131-54.

SILVA, T. S. M. da; COELHO FILHO, M. A.; COELHO, E. F. Boletim meteorológico da estação convencional de Cruz das Almas, BA: variabilidade e tendências climáticas. Cruz das Almas - BA: Embrapa Mandioca e Fruticultura, 2016. Disponívelem:https://www.embrapa.br/mandioca-efruticultura/busca-de-publicacoes/publicacao/1050036/boletim-meteorologico-da-estacaoconvencional-de-cruz-das-almas-ba-variabilidade-etendencias-climaticas.Acesso em: Agosto de 2016.

SOARES, W. A.. Análise comparativa do fluxo de calor no solo em profundidade e na superfície. Revista Brasileira de Geografia Física, v.6, p.665-679, 2013.

WEBER, S., GRAF, A., HEUSINKVELD, B. G. Accuracy of soil heat flux plate measurements in coarse substrates - Field measurements versus a laboratory test. Theoretical and Applied Climatology, v.89, n.1-2,109-114, 2007.

\section{AGRADECIMENTOS}

A FAPESB pela concessão da bolsa de estudo.

Recebido em: 25/04/2020

Aceito para publicação em: 02/10/2020 